\title{
Anti-SARS-CoV-2 Antibodies Within IVIg Preparations: Cross-Reactivities With Seasonal Coronaviruses, Natural Autoimmunity, and Therapeutic Implications
}

OPEN ACCESS

Edited by:

Pier Luigi Meroni,

Istituto Auxologico Italiano (IRCCS), Italy

Reviewed by:

Tadej Avcin

University Medical Centre Ljubljana, Slovenia

Stephen Rawlings,

University of California, San Diego, United States

${ }^{*}$ Correspondence:

Marinos C. Dalakas

marinos.dalakas@jefferson.edu;

mdalakas@med.uoa.gr

Specialty section:

This article was submitted to Autoimmune and Autoinflammatory Disorders,

a section of the journal

Frontiers in Immunology

Received: 08 November 2020 Accepted: 01 February 2021

Published: 17 February 2021

Citation:

Dalakas MC, Bitzogli K and Alexopoulos $H$ (2021) Anti-SARS-CoV-2 Antibodies Within IVIg Preparations: Cross-Reactivities With Seasonal Coronaviruses, Natural Autoimmunity, and Therapeutic Implications.

Front. Immunol. 12:627285. doi: 10.3389/fimmu.2021.627285

\begin{abstract}
Marinos C. Dalakas ${ }^{1,2 *}$, Kleopatra Bitzogli ${ }^{2}$ and Harry Alexopoulos ${ }^{2}$
${ }^{1}$ Department of Neurology, Thomas Jefferson University, Philadelphia, PA, United States, ${ }^{2}$ Neuroimmunology Unit, National and Kapodistrian University of Athens Medical School, Athens, Greece
\end{abstract}

Introduction: Cross-reactivity to SARS-CoV-2 antigenic peptides has been detected on T-cells from pre-pandemic donors due to recognition of conserved protein fragments within members of the coronavirus's family. Further, preexisting antibodies recognizing SARS-CoV-2 with conserved epitopes in the spike region have been now seen in uninfected individuals. High-dose Intravenous Immunoglobulin (IVIg), derived from thousands of healthy donors, contains natural IgG antibodies against various antigens which can be detected both within the IVIg preparations and in the serum of IVIg-receiving patients. Whether IVIg preparations from pre-pandemic donors also contain antibodies against pre-pandemic coronaviruses or autoreactive antibodies that cross-react with SARS-CoV-2 antigenic epitopes, is unknown.

Methods: 13 samples from 5 commercial IVIg preparations from pre-pandemic donors (HyQvia (Baxalta Innovations GmbH); Privigen (CSL Behring); Intratect (Biotest AG); IgVena (Kedrion S.p.A); and Flebogamma (Grifols S.A.) were blindly screened using a semi-quantitative FDA-approved and validated enzyme-linked immunosorbent assay (ELISA) (Euroimmun, Lubeck, Germany).

Results: Nine of thirteen preparations (69.2\%), all from two different manufactures, were antibody-positive based on the defined cut-off positivity (index of sample OD to calibrator $\mathrm{OD}>1.1)$. From one manufacturer, $7 / 7$ lots (100\%) and from another $2 / 3$ lots $(67 \%)$, tested positive for cross-reacting antibodies. $7 / 9$ of the positive preparations (77\%) had titers as seen in asymptomatically infected individuals or recent COVID19-recovered patients, while 2/9 (23\%) had higher titers, comparable to those seen in patients with active symptomatic COVID-19 infection (index > 2.2).

Conclusion: Pre-pandemic IVlg donors have either natural autoantibodies or pre-pandemic cross-reactive antibodies against antigenic protein fragments conserved among the "common cold" - related coronaviruses. The findings are important in: (a) assessing true anti-SARS-CoV-2-lgG seroprevalence avoiding false positivity in IVlg-receiving patients; (b) exploring potential protective benefits in patients with 
immune-mediated conditions and immunodeficiencies receiving acute or chronic maintenance IVIg therapy, and (c) validating data from a recent controlled study that showed significantly lower in-hospital mortality in the IVlg- treated group.

Keywords: IVIg, antibodies to SARS-CoV-2, autoreactivity, cross-reactivity with COVID-19, COVID-19

\section{INTRODUCTION}

Efficient immune surveillance of infected or recovering populations from COVID-19 is important in the fight against the pandemic. Recent evidence suggests that anti-SARS-CoV-2 immunity not only occurs after an active infection, but may also precede an infection. Cross-reactivity to SARS-CoV-2 antigenic peptides has been detected on T-cells and B-cells from pre-pandemic donors owing to recognition of protein fragments conserved among common cold-related coronaviruses $(1,2)$. Spike protein-reactive CD4+ T cells are not only seen in $83 \%$ of patients with COVID-19 infection but also in 35\% of healthy donors; further, spike-protein-reactive $\mathrm{T}$ cell lines from healthy donors respond similarly to the C-terminal region of the spike proteins of both, the human endemic coronaviruses as well as that of SARS-CoV-2 (2). Most importantly, antibodies cross-reacting to SARS-CoV-2 have not only been detected in healthy individuals (3) but, based on just published evidence, preexisting antibodies recognizing SARS-CoV-2 in uninfected individuals have conserved epitopes in the spike region targeted by neutralizing antibodies (4).

Intravenous Immunoglobulin (IVIg), derived from thousands of healthy donors, contains natural and cross-reactive IgG autoantibodies against various antigens at various thresholds of detection owing to accumulation of low antibody concentrations from single individuals. These antibodies can be detected at clinically significant levels not only within the IVIg preparations but also in the serum of IVIg-receiving patients (5). Whether IVIg preparations also contain antibodies that may crossreact with SARS-CoV-2 antigenic epitopes, is unknown. The information is important in accurately assessing anti-SARSCoV-2-IgG seroprevalence, to avoid false positivity in IVIgreceiving patients, but also in exploring potential protective benefits in patients with immune-mediated conditions and immunodeficiencies receiving acute and chronic maintenance therapy (6).

\section{METHODS}

We examined if various commercial IVIg preparations contain anti-SARS-CoV-2-antibodies, due to cross-reactivity with prepandemic anti-coronavirus IgG antibodies or the existence of natural IgG antibodies, and accurately measured antibody titers to assess if they are clinically meaningful, compared to titers seen in COVID-19 infected patients.

We tested 13 samples from 5 commercial IVIg preparations using a semi-quantitative FDA-approved, enzyme-linked immunosorbent assay (ELISA) (Euroimmun, Lubeck, Germany) that measures anti-SARS-CoV-2-antibodies directed against the
S1 viral spike protein. The assay, according to the manufacturer, has a $98.5 \%$ sensitivity and $99 \%$ specificity. An independent serological survey has validated this method reporting $93 \%$ sensitivity and $100 \%$ specificity (7). We have also used the same assay to screen serum and CSF from COVID-19 infected patients with encephalpathy (8) as well as COVID19 recovered patients and uninfected health-care workers (9). The cut-off positivity is set as the $\mathrm{OD}$ value measured at $450 \mathrm{~nm}$ divided by the $\mathrm{OD}$ value of the provided calibrator, being $>1.1$.

The following IVIg preparations were screened: HyQvia (Baxalta Innovations $\mathrm{GmbH}$ ); Privigen (CSL Behring); Intratect (Biotest AG); IgVena (Kedrion S.p.A); and Flebogamma (Grifols S.A.). From one brand, 7 different lots were available and from another one 3 different lots. A total of 13 lots were screened. All preparations were coded by manufacturer and lot (A17, B1-3, C, D, E) and used as liquid preparations in either 1:250 or 1:500 dilution to match normal human serum IgG concentration $(0.5 \mathrm{mg} / \mathrm{ml})$. No dissolution was needed ensuring the lack or aggregate formation. No colloids were added as all IVIg preparations tested contained protective colloids.

Control sera were used from patients with various autoimmune neurological diseases, autoimmune neuropathies and non-COVID19 ICU-hospitalized patients from the serum Biobank of the Department of Pathophysiology, University of Athens Medical School. Ethical approval for Bio-banking had been granted from the University of Athens Ethics Committee.

\section{RESULTS}

Nine of thirteen preparations (69.2\%), all from 2 different manufactures, tested positive based on the manufacturer's cut-off (index of sample OD to calibrator OD > 1.1) (Table 1). From one manufacturer, all 7 lots (100\%) were positive (A1-A7-Table 1); from another, $2 / 3(67 \%)$ lots (B1, B2) were positive while the third lot (B3-Table 1) was borderline (index 1.08) and we called it negative. Among the positive preparations, 7/9 (77\%) had titers as seen in asymptomatically infected individuals or recent COVID19-recovered patients $(8,9)$ while $2 / 9(23 \%)$ had higher titers with index $>2$ (2.32 and 2.21 respectively), comparable to those seen in patients with active symptomatic COVID-19 infection as concurrently measured in the sera of 50 COVID19 PCR-confirmed symptomatic patients (index range 1.19-8.47) $(8,9)$.

To further validate our results, we tested as controls prepandemic archived sera from: (a) 49 neurological patients with autoimmune neuropathies; only one had a titer of 2.32, comparable to COVID-19 infected patients and the IVIgpositive preparations (whether this patient had been previously receiving immunotherapy with IVIg is however unknown); and 
TABLE 1 | IVlg preparations and antibody titers.

\begin{tabular}{lcc}
\hline IVIg preparations & Concentration of IgG & Anti-SARS-CoV-2 titers \\
\hline A1 & $100 \mathrm{mg} / \mathrm{ml}$ & 1.46 - POSITIVE \\
A2 & $100 \mathrm{mg} / \mathrm{ml}$ & $1.28-$ POSITIVE \\
A3 & $100 \mathrm{mg} / \mathrm{ml}$ & $1.41-$ POSITIVE \\
A4 & $100 \mathrm{mg} / \mathrm{ml}$ & $1.27-$ POSITIVE \\
A5 & $100 \mathrm{mg} / \mathrm{ml}$ & $1.40-$ POSITIVE \\
A6 & $100 \mathrm{mg} / \mathrm{ml}$ & $2.21-$ POSITIVE \\
A7 & $100 \mathrm{mg} / \mathrm{ml}$ & $2.32-$ POSITIVE \\
B1 & $50 \mathrm{mg} / \mathrm{ml}$ & $1.38-$ POSITIVE \\
B2 & $50 \mathrm{mg} / \mathrm{ml}$ & $1.18-$ POSITIVE \\
B3 & $50 \mathrm{mg} / \mathrm{ml}$ & $1.08-$ NEGATIVE \\
C & $100 \mathrm{mg} / \mathrm{ml}$ & $0.89-$ NEGATIVE \\
D & $50 \mathrm{mg} / \mathrm{ml}$ & $0.66-$ NEGATIVE \\
E & $50 \mathrm{mg} / \mathrm{ml}$ & $0.74-$ NEGATIVE \\
\hline
\end{tabular}

(b) 30 ICU-hospitalized patients; all were anti-SARS-CoV-2 antibody negative.

\section{DISCUSSION}

IVIg preparations, made from pre-pandemic healthy donors, exhibit cross reactivities with SARS-CoV-2 S1 antigens, with some preparations having clinically significant antibody titers comparable to those seen in COVID-19 infected patients. The findings suggest that the respective healthy IVIg donors had either natural autoantibodies or cross-reactive antibodies against antigenic protein fragments conserved among the other "common cold" -related coronaviruses, similar to the cross-reactivity observed for T-cells and B cells from unexposed donors $(1,2)$.

Being cognizant about false positivity, we ensured the reliability of the anti-SARS-CoV-2- antibody ELISA method by: (a) using an FDA-approved assay, same as in other major serological surveys (7-9); (b) testing pre-COVID-19 sera from patients with autoimmune diseases which are often polyreactive; and (c) testing 50 PCR-confirmed COVID-19 infected patients.

IVIg has been effectively used in some COVID-19 patients including COVID-19- immune inflammatory syndrome resembling Kawasaki's disease (10) but whether the antibodies detected in the present study have any protective or therapeutic effects in COVID-19-exposed populations is uncertain. A recent randomized placebo-controlled double-blind clinical trial with IVIg in severe COVID-19-infected patients showed that the in-hospital mortality rate was significantly lower in the IVIg group compared to the control group (11). Considering that acquired immunity may be short-lived in persons with mild or asymptomatic COVID-19 infection (12), the IVIg administrations currently prescribed as monthly maintenance for patients with immunodeficiency and immune-mediated diseases (6), may theoretically provide some added benefit, even though these natural or cross-reactive antibodies are not affinity maturated for SARS-CoV-2. Of interest, some of our elderly IVIgreceiving patients with autoimmune neuropathies have been reporting in follow-up visits that did well when infected by COVID-19, but we have no objective data to support any IVIg-derived benefit.

The just published evidence that preexisting seasonal coronavirus antibodies in uninfected individuals cross-react with SARS-CoV-2 recognizing conserved epitopes in the spike region targeted by neutralizing antibodies, may have important ramification for natural infection (4) and the potential role of IVIg. Because prior immunity induced by seasonal coronavirus can reduce the transmission of homologous and heterologous strains (4), enhancing the preexisting seasonal coronavirus-elicited immunity with the antibodies within the IVIg preparations may have an impact on the natural course of SARS-CoV-2 infection. Such a potential effect of IVIg may be also enhanced by the known additional multilevel actions of IVIg in autoimmunity, including cytokine neutralization, immunoregulatory $\mathrm{T}$ cells, co-stimulatory molecules, MHC-expression, complement and natural autoantibodies $(6,13)$.

Apart from a potential benefit, the observations are also important in assessing SARS-CoV-2 seroprevalence, emphasizing the need to ensure that sera from prior IVIg-treated patients may contain SARS-CoV-2-antibodies derived from IVIg rather than form acquired antiviral humoral immunity.

It would be of interest in a future study to check for antibodies against the S2 subunit when kits with verified reliability become available. Most importantly, it will be relevant to examine if the noted anti-SARS-CoV-2 antibodies have a neutralizing activity.

\section{DATA AVAILABILITY STATEMENT}

The original contributions presented in the study are included in the article/supplementary material, further inquiries can be directed to the corresponding author/s.

\section{AUTHOR CONTRIBUTIONS}

All authors contributed to conception and execution of the work, writing, and reviewing the manuscript.

\section{FUNDING}

This work was supported by Thomas Jefferson University and University of Athens. 


\section{REFERENCES}

1. Le Bert N, Tan AT, Kunasegaran K, Tham CYL, Hafezi M, Chia A, et al. SARS-CoV-2-specific $T$ cell immunity in cases of COVID19 and SARS, and uninfected controls. Nature. (2020) 584:457-62. doi: 10.1038/s41586-020 2550-z

2. Braun, Loyal L, Frentsch M, Hippenstiel S, Dingeldey M, Kruse B, et al. SARS-CoV-2-reactive T cells in healthy donors and patients with COVID-19. Nature. (2020) 587:270-4. doi: 10.1038/s41586-020-2598-9

3. Sood N, Simon P, Ebner P, Eichner D, Reynolds J, Bendavid E, et al. Seroprevalence of SARS-CoV-2-specific antibodies among adults in Los Angeles County, California, on April 10-11, 2020. JAMA. (2020) 323:2425-7. doi: 10.1001/jama.2020.8279

4. Ng KW, Faulkner N, Cornish GH, Rosa A, Harvey R, Hussain S, et al. Preexisting and de novo humoral immunity to SARS-CoV-2 in humans. Science. (2020) 370:1339-43. doi: 10.1126/science.abe1107

5. Dimitriadou MM, Alexopoulos H, Akrivou S, Dalakas MC. Antineuronal antibodies within the IVIg preparations: importance in clinical practice. Neurotherapeutics. (2020) 17:235-42. doi: 10.1007/s13311-019-0 0796-3

6. Dalakas MC. Intravenous immunoglobulin in autoimmune neuromuscular diseases. JAMA. (2004) 291:2367-75. doi: 10.1001/jama.291.19.2367

7. Stringhini S, Wisniak A, Piumatti G, Azman AS, Lauer SA, Baysson H, et al. Seroprevalence of anti-SARS-CoV-2 IgG antibodies in Geneva, Switzerland (SEROCoV-POP): a population-based study. Lancet. (2020) 396:313-9. doi: 10.1016/s0140-6736(20)31304-0

8. Alexopoulos H, Magira E, Bitzogli K, Kafasi N, Vlachoyiannopoulos P, Tzioufas A, et al. Anti-SARS-CoV-2 antibodies in the CSF, bloodbrain barrier dysfunction and neurological outcome: studies in 8 comatose patients. Neurol Neuroimmunol Neuroinflamm. (2020) 7:e893. doi: 10.1212/NXI.0000000000000893
9. Vlachoyiannopoulos P, Alexopoulos H, Apostolidi I, Bitzogli K, Barba C, Athanasopoulou E, et al. Anti-SARS-CoV-2 antibody detection in healthcare workers of two tertiary hospitals in Athens, Greece. Clin Immunol. (2020) 221:108619. doi: 10.1016/j.clim.2020

10. Lee PY, Day-Lewis M, Henderson LA, Friedman KG, Lo J, Roberts JE, et al. Distinct clinical and immunological features of SARS-COV-2-induced multisystem inflammatory syndrome in children. J Clin Invest. (2020) 130:5942-50. doi: 10.1172/JCI141113

11. Gherebaghi N, Nejadrahim R, Mousavi SJ, Sadat-Ebrahimi SR, Hajizadeh R. The use of intravenous immunoglobulin gamma for the treatment of severe coronavirus disease 2019: a randomized placebo-controlled double-blind clinical trial. BMC Infectious Diseases. (2020) 20:786. doi: 10.1186/s12879-020-05507-4

12. Long QX, Tang XJ, Shi QL, Li Q, Deng H-J, Yuan J, et al. Clinical and immunological assessment of asymptomatic SARS-CoV-2 infections. Nat Med. (2020) 26:1200-4. doi: 10.1038/s41591-020-0965-6

13. Kaveri SV. Intravenous immunoglobulin: exploiting the potential of natural antibodies. Autoimmun Rev. (2012) 11:792-4. doi: 10.1016/j.autrev.2012.02.006

Conflict of Interest: The authors declare that the research was conducted in the absence of any commercial or financial relationships that could be construed as a potential conflict of interest.

Copyright (C) 2021 Dalakas, Bitzogli and Alexopoulos. This is an open-access article distributed under the terms of the Creative Commons Attribution License (CC BY). The use, distribution or reproduction in other forums is permitted, provided the original author(s) and the copyright owner(s) are credited and that the original publication in this journal is cited, in accordance with accepted academic practice. No use, distribution or reproduction is permitted which does not comply with these terms. 